\title{
Soziologie des Alterns
}

\author{
Janis Detert
}

Online publiziert: 23. März 2020

(C) Der/die Autor(en) 2020

Hank, Karsten, Frank Schulz-Nieswandt, Michael Wagner und Susanne Zank(Hrsg.):

Alternsforschung. Handbuch für Wissenschaft und Praxis. Baden-Baden: Nomos Verlagsgesellschaft 2019. 696 Seiten. ISBN: 978-3-8478-3328-6. Preis: $€ ~ 58,-$.

Karsten Hank, Frank Schulz-Nieswandt, Michael Wagner und Susanne Zank haben sich der Herausforderung angenommen, einen Überblick über die deutschsprachige Alternsforschung zusammenzustellen. Die Sozialforschung über Prozesse des Alterns und das Leben im Alter ist mittlerweile ausdifferenziert und entsprechend unübersichtlich, sodass Wissenschaft und Praxis froh sein können, auf 696 Seiten aktuelle Einblicke präsentiert zu bekommen.

Auf die kurze Einleitung folgen 26 Beiträge, die von den Herausgebern ,klassischen“ und ,neuen“ Themen und Perspektiven der Alternsforschung zugeteilt sind. Im ,klassischen“ ersten Teil befassen sich dann aber jeweils mehrere aufeinanderfolgende Texte mit einem übergreifenden Themenfeld. So folgen auf den die Einleitung ergänzenden kurzen Text von Schulz-Nieswandt vier Aufsätze, die das gesamte Handbuch fundieren: Josef Ehmer gibt einen Überblick über „,Altersbilder und Konzeptionen des Alter(n)s im historisch-kulturellen Vergleich“. Er konstatiert für die jüngere Gegenwart, dass wegen der ,wachsenden Zahl von Akteuren im Altersdiskurs [...] die Ambivalenz von Altersbildern und die Vielstimmigkeit von Altersdiskursen auch in der Gegenwart nicht verblasst" sind (S. 33 f.).

Als zentrale Funktion der Altersbilder wird mit Blick auf Europa und Ostasien festgestellt: „Sie [die Altersbilder] dienten und dienen nicht nur zur kulturellen Bewältigung der Endlichkeit des Lebens, sondern mehr noch der Regulierung von

\footnotetext{
J. Detert $(\bowtie)$

Institut für Soziologie, Ludwig-Maximilians-Universität München

Konradstraße 6, 80801 München, Deutschland

E-Mail: janis.detert@soziologie.uni-muenchen.de
} 
Generationenbeziehungen“(S. 41). Der Beitrag erfüllt eine zentrale Funktion, indem er die wenigen historischen und kulturübergreifenden Konstanten der Lebensphase Alter aufzeigt, insbesondere aber die Veränderbarkeit von Altersbildern betont. Clemens Tesch-Römer diskutiert anschließend acht ausgewählte ,Theorien der sozialund verhaltenswissenschaftlichen Alternsforschung"und formuliert dazu sechs zentrale Aspekte, mit denen sich entsprechende Theorien befassen: „Altern als Teil des Lebenslaufs, Bedingungen des Alterns, Altern im Kontext, Vielfalt und Ungleichheit des Alterns, Facetten des Alterns sowie die Möglichkeit von Interventionen für ein gutes Leben im Alter“ (S. 49). Erwartungsgemäß beginnt er mit der Disengagement-Theorie, die zwar empirisch nicht haltbar, aber nach wie vor wertvoll sei, nicht zuletzt, weil sie ,ein Gegengewicht zur bisweilen allzu optimistischen Grundhaltung der ,positiven Gerontologie“"“ (S. 57) darzustellen vermag. Klotz und Simm geben insbesondere für Sozialwissenschaftler eine bereichernde Ergänzung, indem sie detailreich in die „Biologie des Alterns“ einführen und herausarbeiten, wieviel Forschungsbedarf auch hier noch besteht. So sei beispielsweise die Messung des biologischen Alters „schwieriger als gemeinhin angenommen“ (S. 103). Daraufhin erläutern Wagner und Geithner die „Lebenslaufperspektive“ anhand des Beispiels kultureller Aktivitäten im Alter. In vielen der folgenden Beiträge zeigt sich, wie bedeutend diese Perspektive in der aktuellen Alternsforschung ist.

Auf den programmatischen Beginn folgen drei Beiträge zu sozioökonomischen Aspekten des Alterns: Während Fachinger ein zuversichtliches Szenario der deutschen Alterssicherung gerade wegen der Verzahnung von Umlage- und kapitalfundierten Verfahren zeichnet, sind Simonson und Vogel skeptischer und folgen in ihrem Beitrag zu „Aspekten sozialer Ungleichheit im Alter“ der Kontinuitätstheorie, nach der Ungleichheiten im Alter stabil sind oder gar verstärkt werden. Heinze und Schneider betonen die „Sozioökonomischen Potenziale des Alters“ und weisen u. a. auf die hohe Kaufkraft vieler älterer Konsumenten hin. Vier Beiträge fokussieren anschließend auf die Themen Gesundheit, Medizin, Pflege und Lebensende, erwartungsgemäß stärker aus (sozial-)psychologischer und pflegwissenschaftlicher Perspektive. Hier zeigt sich deutlich, dass die Beforschung der ,,alten Alten“ gerade erst richtig beginnt. Drei Beiträge über den Nahbereich älterer Menschen schließen die ,klassischen“ Themen ab: Ellwardt und Hank plädieren für eine netzwerktheoretische Herangehensweise, Klaus und Mahne betrachten „Partnerschaft und Familie im Alter" und Ehrlinghausen und Hank thematisieren die „Gesellschaftliche Teilhabe Älterer in der Nacherwerbsphase“.

Im zweiten, „neuen“ Teil des Handbuchs stehen die 11 Beiträge jeweils stärker für sich. Penger, Oswald und Wahl diskutieren zu Beginn Wohnumfeld und Mobilitätsverhalten älterer Menschen. Im Anschluss thematisieren Wilhelm und Zank „Altern im historischen Kontext Deutschlands“. Anders als der Titel nahelegt, geht es den Autoren um Kriegs-, Vertreibungs- und Verfolgungserfahrungen, auf deren traumatische Folgen Pflegeeinrichtungen vorbereitet sein sollten. Dies verdeutlicht, wie wichtig die Lebenslaufperspektive in der Arbeit mit älteren Menschen ist. Die zwei nachfolgenden Beiträge behandeln „Gewalterfahrungen älterer Menschen im sozialen Nahraum“ sowie „Alter(n) und Straffälligkeit“. Dass ältere Menschen besonders vulnerabel sind, erstaunt leider nicht; dass Strafanstalten allerdings im Zuge demografischer Alterung umtreibt, wie mit der ,,stark wachsenden Zahl älterer Ge- 
fangener" umzugehen ist (S. 509), weist auf ein neues Problemfeld hin. Brose und Zank gehen der These nach, dass nicht das Interesse an, sondern Möglichkeit und Gelegenheit zu „Sexualität im Alter“ abnehmen. Wenngleich diese These auf Lebenslauf und Biografie älterer Menschen abzielt, bleiben diese Perspektiven im dennoch aufschlussreichen Beitrag wenig beachtet. Schmidt und Wahl prognostizieren, „dass der Einsatz von Technik für ein erfolgreiches Altern vielfältige Potenziale bietet" (S. 539). Potenziale, nicht zuletzt für Forschende, die sich neue Daten(mengen) erhoffen. Mit Blick auf „Ältere Migrantinnen und Migranten in Deutschland“ ist die Datenlage „mehr als nur unbefriedigend“ (S. 573). Dennoch kommt Steinbach in ihrem instruktiven Beitrag über die ältesten Generationen der ehemaligen Arbeitsmigranten und (Spät-)Aussiedler zu dem Schluss, dass diese ,,in der Bundesrepublik Deutschland eine besonders vulnerable Gruppe darstellen“ (S. 574). Neise et al. wenden sich „Lebensqualitäten in der Hochaltrigkeit“ zu und zeigen, dass diese Lebensphase ,,von einer Gleichzeitigkeit von Entwicklungspotenzialen und Vulnerabilität gekennzeichnet [ist], wobei sich gerade in der Verletzlichkeit des Lebens Entwicklungspotenziale zeigen können“ (S. 591). Jahnsen und Woopen zeigen, dass Spiritualität ,nicht dem Alter vorbehalten und auch nicht nur für die Mehrzahl der älteren Menschen von besonderer Relevanz" ist (S. 624). Branzke et al. denken über eine „Ethik des Alterns“ nach, was forschungsethische Fragen miteinschließt. Auch mit Blick auf die Alternsforschung fordern sie eine verstärkte Diskussion des „gerechten“ Alter(n)s und knüpfen an Positionen an, die sich kritisch mit ,erfolgreichen“ und „aktiven“ Altern befassen. Zum Abschluss des Handbuchs betonen Schlomann und Rietz im Überblick über die ,Erhebung von Daten in der älteren Bevölkerung“ mit Blick auf datenintensive Forschungsprojekte, ,dass auch repräsentative Befragungen älterer Personen funktionieren und $\mathrm{zu}$ validen Ergebnissen führen, wenn durchdachte Erhebungsdesigns verwendet werden“ (S. 676).

Die Breite der Themen im vorliegenden Handbuch ist beachtlich und dank der durchgängig hohen Qualität der Beiträge dürfte es sich in Forschung, Lehre und gewiss auch in diversen Praxisfeldern bewähren. Allerdings ist bedauerlich, dass die Herausgeber das Handbuch nicht stärker strukturiert haben. Lediglich „klassische“ von „neuen“ Themen und Perspektiven zu unterscheiden ist oberflächlich, nicht in jedem einzelnen Fall naheliegend und bietet nicht die Orientierung, die ein Handbuch geben könnte. Der Verzicht auf eine ausdrücklichere Strukturierung erstaunt, sind Auswahl und Anordnung im ersten Teil doch keineswegs willkürlich. Vielmehr werden Kategorien erkennbar, zu denen auch die Texte im zweiten Teil gepasst hätten: programmatische Überlegungen, sozioökonomische Aspekte, Körper und Gesundheit, individuelles Umfeld. Eine Gliederung entlang dieser - oder anderer - inhaltlich begründeter Kategorien hätte gerade denjenigen geholfen, für die Alternsforschung Neuland ist.

Die sehr knappe Einleitung weist zudem darauf hin, dass sich die Herausgeber mit allzu deutlichen Eingriffen zurückhalten wollten. Dies hat einerseits die positive Folge, dass alle Texte auch außerhalb des Handbuchkontextes funktionieren. Allerdings muss man andererseits diskutieren, weshalb insbesondere kritische Gerontologie und qualitative Forschungsmethoden nicht eigens gewürdigt wurden. So erscheint die Alternsforschung hier methodologisch einseitiger als sie ist. Erkennbar wird das exemplarisch an der vielfachen Betonung von „Potenzialen“ des Alter(n)s, 
wobei nicht geklärt wird, was darunter zu verstehen ist. Wer darüber hinwegsehen kann, findet im Handbuch Alternsforschung aktuelle, gründliche und durchweg anregende Beiträge.

Funding Open Access funding provided by Projekt DEAL.

Open Access Dieser Artikel wird unter der Creative Commons Namensnennung 4.0 International Lizenz veröffentlicht, welche die Nutzung, Vervielfältigung, Bearbeitung, Verbreitung und Wiedergabe in jeglichem Medium und Format erlaubt, sofern Sie den/die ursprünglichen Autor(en) und die Quelle ordnungsgemäß nennen, einen Link zur Creative Commons Lizenz beifügen und angeben, ob Änderungen vorgenommen wurden.

Die in diesem Artikel enthaltenen Bilder und sonstiges Drittmaterial unterliegen ebenfalls der genannten Creative Commons Lizenz, sofern sich aus der Abbildungslegende nichts anderes ergibt. Sofern das betreffende Material nicht unter der genannten Creative Commons Lizenz steht und die betreffende Handlung nicht nach gesetzlichen Vorschriften erlaubt ist, ist für die oben aufgeführten Weiterverwendungen des Materials die Einwilligung des jeweiligen Rechteinhabers einzuholen.

Weitere Details zur Lizenz entnehmen Sie bitte der Lizenzinformation auf http://creativecommons.org/ licenses/by/4.0/deed.de.

Janis Detert M.A., Wissenschaftlicher Mitarbeiter am Institut für Soziologie der Ludwig-MaximiliansUniversität München. Forschungsschwerpunkte: Historische Soziologie, Soziologie des Vergleichens, Soziologie der Generationen, Soziologie des Alter(n)s. Aktuelle Publikation: Fußball als Massenphänomen. Zur Soziologie einer Sportart. Schulmagazin 5-10, 4, 2016. 